\title{
Controversies surrounding female athletes with differences in sexual development
}

\author{
Ahmed Khattab, ${ }^{1}$ Ian Marshall, ${ }^{1}$ and Sally Radovick' \\ 'Department of Pediatrics, Child Health Institute of New Jersey, Rutgers-Robert Wood Johnson Medical School, Rutgers, the State University of New Jersey, New Brunswick, New Jersey, USA.
}

\section{Regulations and testosterone levels: implications for the female athlete}

Sports-governing authorities currently acknowledge that not all disorders of sexual development (DSDs), now often referred to as differences in sexual development, offer a competitive advantage to the affected female athlete. The advantages appear to be related to the degree of functional androgen levels in these athletes. In this way, testosterone levels have been used as a determinant of ineligibility for certain competitions and implemented as a metric to disqualify female athletes in women's sports. Conversely, testosterone levels have not been used as a qualifier for participation in male competitions. These parameters have been directly included in policies by different sporting bodies. For example, in 2018, the International Association for Athletics Federation (IAAF), which governs track and field competitions, issued regulations banning female athletes with testosterone levels above a certain threshold from participating in the 400-, 800-, and 1500-meter races. This decision was based on the assumption that higher testosterone levels may offer an unfair advantage to these athletes. This decision is clearly biased against individuals with disorders of androgen insensitivity, since their testosterone levels would be significantly elevated but nonfunctional.

These types of policies and regulations may force athletes to turn to medical or surgical solutions to lower their naturally occurring testosterone levels to enable their participation in female-only competitions. One particularly infamous case in recent years is that of Caster Semenya, a South African runner and former Olympic gold medalist. Semenya was revealed to have a DSD, but her exact condition and testosterone levels were not made pub- lic. Although she was previously allowed to compete as a woman, the new IAAF regulations exclude her from competing. Despite taking legal action, Semenya lost her appeal in the court for arbitration for sports. Although the court declared that Semenya had not done anything wrong, and that the case was not about cheating, the ruling was based on the protection of women-only sports. The new IAAF regulations have forced Semenya and other athletes in similar positions to undergo unwanted investigations and medical interventions to circumvent their exclusion from participation in their sport of choice $(1,2)$.

In this Viewpoint, we address the controversy involving female athletes who are thought to derive a competitive advantage due to higher androgen levels relative to their competitors. DSDs - conditions with atypical chromosomal, gonadal, or anatomic sex development - are not to be confused with the transgender state, a condition with incongruence between the assigned sex and the experienced gender of an individual who seeks to change sexual characteristics through medical intervention $(3,4)$.

\section{Testosterone (the anabolic steroid) and sports}

Testosterone is an androgenic steroid that increases muscle mass, strength, and recovery (Figure 1). Testosterone increases erythropoiesis and hence the athlete's oxygen-carrying capacity. Androgens are also believed to be sports enhancing due to their positive effects on the central nervous system (CNS), including increased excitability and regeneration of motor neurons, as well as aggressiveness and risk taking, behavioral traits that have been suggested to be beneficial in sports. Hence, the edge that males are assumed

Conflict of interest: The authors have declared that no conflict of interest exists.

to have over females in sports is primarily related to testosterone levels (5).

The above-mentioned advantages explain why testosterone is the most common illicit performance-enhancing drug used by female athletes. However, the advantages of endogenously occurring androgens are unclear and remain debatable in the literature. For example, an extensive review by Ferguson-Smith et al. concluded that endogenous hyperandrogenemia does not offer female athletes any athletic advantage (6).

Nevertheless, whether or not female athletes affected by DSDs have a competitive advantage is a controversial subject. In past decades, there have been extensive debates on the eligibility of athletes with what has been referred to as either naturally occurring or pathophysiologically increased testosterone levels to participate in competitive sports, and the perceived fairness of their participation in women's sports (4).

\section{The underlying etiology associated with DSDs}

DSDs in female athletes include disorders of gonadal development, excess androgen production in individuals with a $46, \mathrm{XX}$ karyotype (typical female karyotype), and defects resulting in impaired androgen synthesis or action in those with a $46, \mathrm{XY}$ karyotype (typical male karyotype).

Significant phenotypic variability is described with ovotesticular DSD (OT-DSD), a rare condition in which the gonad (Ovo-testes) is histologically composed of ovarian follicles and testicular tissue. Of note, the majority of individuals with OT-DSD have a 46, XX karyotype, although association with a 46, XY karyotype has been reported. In 46, XX/XY, XO/ $\mathrm{XY}$ gonadal dysgenesis and $46, \mathrm{XY}$ pure gonadal dysgenesis (partial type), there is a wide spectrum of androgen effects in the phenotypic female (7-9).

Excess androgen production in individuals with a 46, XX karyotype leads to variable degrees of masculinization. Adre- 


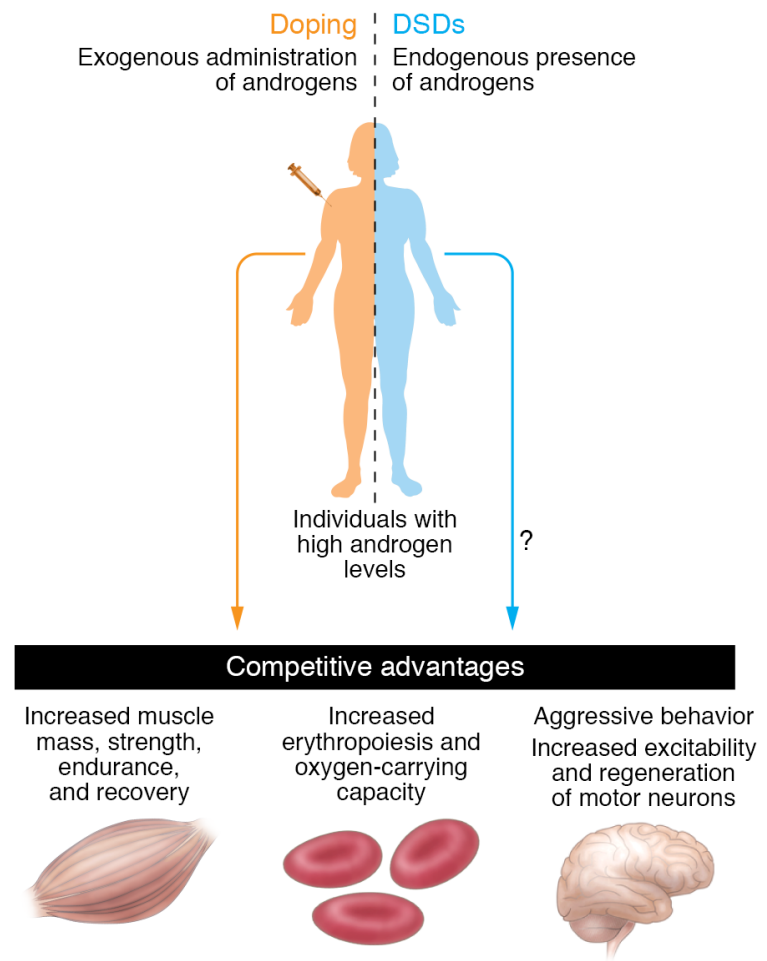

Figure 1. Anabolic effects of testosterone. The anabolic effects of testosterone on the musculoskeletal system, erythropoiesis, central nervous system, and aggressive behavioral traits are well established. The exogenous administration of testosterone and some DSDs are associated with higher testosterone levels. The competitive advantage offered by increased testosterone levels in individuals with DSDs remains debatable.

nal androgen overproduction occurs in congenital adrenal hyperplasia (CAH) due to variable enzymatic deficiencies, such as 21-hydroxylase deficiency CYP21A2 (6p21.33), 33-hydroxysteroid dehydrogenase 2 HSD3B2 (1p12), and $11 \beta$-hydroxylase deficiency CYP11B1 (8q24.3) (10). In adrenal hyperandrogenism, which is associated with these enzymatic deficiencies, the impaired cortisol synthesis leads to interruption of the cortisol-adrenocorticotropic hormone negative feedback system and a secondary increase in adrenocorticotropic hormone levels, with subsequent shunting of adrenal steroidogenesis to the intact androgen pathway (11). Ovarian hyperandrogenism associated with insulin resistance occurs in the most common reproductive disorder polycystic ovarian syndrome (12).

Impaired androgen biosynthesis may be secondary to enzymatic deficiencies (such as side chain cleavage enzyme CYP11A1 [15q24.1], 17-hydroxylase/17,20-lyase CYP17A1 [10q24.32], lipoid CAH StAR [8p11.23], $5 \alpha$ reductase SRD5A2 [2p23.1], or 17 $\beta$-hydroxysteroid dehydrogenase 3 deficiency HSD17B3 [9q22.32]) (10, 13). Affected individuals with a 46, XY karyotype present with significant variability in phenotype and androgen synthesis.

The 46, XY female with androgen insensitivity due to dysfunctional androgen receptors exhibits high levels of nonfunctioning androgens in complete androgen insensitivity syndrome or partially functioning androgens in partial androgen insensitivity syndromes. The phenotype ranges from that of a typical female phenotype in complete insensitivity, to variable degrees of masculinization in partial insensitivity (13).

Due to policies currently in effect, individuals with DSDs are routinely barred from participating in certain activities based on their sex determination at birth. Additionally, the public scrutiny associated with these cases has amplified the psychosocial stigma associated with sex and gender differences. Critical voices have argued that such rules are discriminatory in nature and stigmatize these athletes rather than empower and celebrate their naturally occurring diversity. Many have pointed out that the regulations bring up questions about the eligibility of individuals with other genetic variations, such as genes promoting growth, lung capacity, muscle power, and endurance, among others. These characteristics are also likely to offer advantages for athletes and by the same token should thus be controlled by similar governing rules. However, most of these other variations are not discriminated against, but are in fact celebrated in many instances.

\section{Conclusions}

These debates have raised significant questions on what ultimately defines eligibility for competition under the available binary categories of female and male. Could these parameters alternatively be chromosomal make up, legal sex, a yetundefined measure of androgen action, or perceived gender identity? Alternatively, perhaps, in order to move beyond exclusionary categories, a new athletic gender could be solely determined by serum testosterone levels, thus leveling the playing field for all athletes regardless of today's social constructions of gender. Yet, elevated nonfunctional testosterone levels in androgen insensitivity syndrome will pose a challenge even in this category, since we can only measure androgen levels in the blood and not at the tissue level, where the androgen effect is expected to occur.

Address correspondence to: Ahmed Khattab, Department of Pediatrics, Child Health Institute of New Jersey, 89 French Street, New Brunswick, New Jersey 08901, USA. Phone: 732.235.9378; ak1684@rwjms.rutgers.edu.

1. Huang G, Basaria S. Do anabolic-androgenic steroids have performance-enhancing effects in female athletes? Mol Cell Endocrinol. 2018;464:56-64.

2. Wikipedia contributors. Caster Semenya. http://en.wikipedia.org/wiki/Caster_Semenya. Accessed February 29, 2020.

3. Shumer DE, Nokoff NJ, Spack NP. Advances in the care of transgender children and adolescents. Adv Pediatr. 2016;63(1):79-102.

4. Lee PA, et al. Global disorders of sex development update since 2006: perceptions, approach and care. Horm Res Paediatr. 2016;85(3):158-180.

5. Nguyen TV, et al. Sex-specific associations of testosterone with prefrontal-hippocampal development and executive function. Psychoneuroendocrinology. 2017;76:206-217.

6. Ferguson-Smith MA, Bavington LD. Natu- 
ral selection for genetic variants in sport: the role of $\mathrm{Y}$ chromosome genes in elite female athletes with 46,XY DSD. Sports Med. 2014;44(12):1629-1634.

7. Kilberg MJ, McLoughlin M, Pyle LC, Vogiatzi MG. Endocrine management of ovotesticular DSD, an index case and review of the literature. Pediatr Endocrinol Rev. 2019;17(2):110-116.

8. Tian Q, He F, Zhou Y, Ge Q. Gender verification in athletes with disorders of sex development. Gynecol Endocrinol. 2009;25(2):117-121.
9. Barseghyan H, Vilain E. Chapter 7. The genetics of ovotesticular disorders of sex development. In: New MI, Lekarev O, Parsa A, Yuen TT, O'Malley BW, Hammer GD, eds. Genetic Steroid Disorders. San Diego, California, USA: Academic Press; 2014:261-263.

10. Khattab A, Narasimhan ML, Macdonald A, Costin G, New M. Chapter 47. Disorders of the adrenal cortex in the fetus and neonate. In: Kovacs CS, Deal CL, eds. Maternal-Fetal and Neonatal Endocrinology. Cambridge, Massachusetts, USA: Academic Press; 2020:813-839.
11. Khattab A, Marshall I. Management of congenital adrenal hyperplasia: beyond conventional glucocorticoid therapy. Curr Opin Pediatr. 2019;31(4):550-554.

12. Wang J, Wu D, Guo H, Li M. Hyperandrogenemia and insulin resistance: The chief culprit of polycystic ovary syndrome. Life Sci. 2019;236:116940.

13. Yatsenko SA, Witchel SF. Genetic approach to ambiguous genitalia and disorders of sex development: What clinicians need to know. Semin Perinatol. 2017;41(4):232-243. 